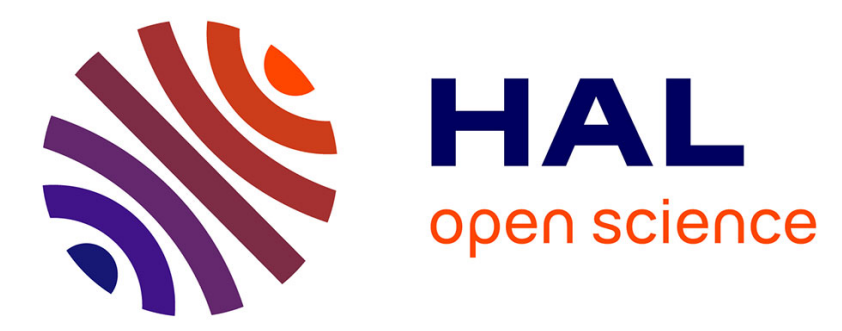

\title{
The lateral patellar retinaculum defect: anatomical study using ultrasound
}

Antoine Moraux, Stefano Bianchi, Francois Tassery, Thomas Le Corroller

\section{To cite this version:}

Antoine Moraux, Stefano Bianchi, Francois Tassery, Thomas Le Corroller. The lateral patellar retinaculum defect: anatomical study using ultrasound. Skeletal Radiology, 2019, 48 (11), pp.1753-1758. 10.1007/s00256-019-03209-w . hal-02534357

\section{HAL Id: hal-02534357 https://hal.science/hal-02534357}

Submitted on 9 Apr 2020

HAL is a multi-disciplinary open access archive for the deposit and dissemination of scientific research documents, whether they are published or not. The documents may come from teaching and research institutions in France or abroad, or from public or private research centers.
L'archive ouverte pluridisciplinaire HAL, est destinée au dépôt et à la diffusion de documents scientifiques de niveau recherche, publiés ou non, émanant des établissements d'enseignement et de recherche français ou étrangers, des laboratoires publics ou privés. 


\title{
The lateral patellar retinaculum defect: anatomical study using ultrasound
}

\author{
Antoine Moraux ${ }^{1,2}$ (D) Stefano Bianchi ${ }^{3} \cdot$ François Tassery $^{4} \cdot$ Thomas Le Corroller $^{5}$
}

\begin{abstract}
Objective The purpose of this study was to evaluate whether ultrasound allows precise assessment of a focal defect at the lateral patellar retinaculum (LPR) and to determine its anatomical significance.

Materials and methods This work was initially undertaken in four cadaveric specimens and followed by high-resolution ultra-sound study in 48 healthy adult volunteers (96 knees) by two musculoskeletal radiologists. Dimension and location of the LPR defect and its relations to adjacent anatomical structures were analyzed.

Results A focal defect of the LPR through which vessels penetrated was constantly identified by ultrasound in our population. The mean transverse diameter of the defect was $2.4 \mathrm{~mm}$ (range, 1-6 mm). The defect was located a mean of 23.7 $\mathrm{mm}$ (range, 18-30 $\mathrm{mm}$ ) proximal to the lateral tibial plateau and $6.6 \mathrm{~mm}$ (range, 4-9 $\mathrm{mm}$ ) lateral to the patellar tendon. Anatomical dissection demonstrated that the LPR defect is related to perforating vessels that originate from the peripatellar anastomotic ring. Conclusions The focal defect of the LPR can be depicted by ultrasonography. Reproducible anatomical landmarks for its detection could be defined. Our data suggest the LPR defect may be considered a normal variant rather than a pathologic change.
\end{abstract}

Keywords Knee $\cdot$ Ultrasound $\cdot$ Patella $\cdot$ Anatomy

\begin{tabular}{ll}
\multicolumn{2}{l}{ Abbreviations } \\
ITT & Iliotibial tract \\
LFC & Lateral femoral condyle \\
LPR & Lateral patellar retinaculum \\
MFC & Medial femoral condyle \\
MPR & Medial patellar retinaculum \\
MR & Magnetic resonance \\
P & Patella \\
PT & Patellar tendon \\
QT & Quadricipital tendon \\
TT & Tibial tuberosity \\
TP & Tibial plateau
\end{tabular}

Antoine Moraux amoraux@yahoo.com

1 Imagerie Médicale Jacquemars Giélée, 73 rue Jacquemars Giélée, 59000 Lille, France

2 Hôpital Privé La Louvière, Ramsay Générale de Santé, 59000 Lille, France

3 CIM SA Cabinet Imagerie Médicale, CH 1208 Geneva, Switzerland

4 Fédération Française de Basketball, 75013 Paris, France

5 Hôpital Sainte Marguerite, 13009 Marseille, France
US Ultrasonography
VM Vastus medialis
VL Vastus lateralis

\section{Introduction}

The lateral patellar retinaculum (LPR) is a complex structure that acts as a passive stabilizer of the femoropatellar joint. Anatomically, the LPR is a two-layered fibrous structure with a superficial layer attached medially to the patella and patellar tendon and extending laterally to the iliotibial band, and a deep layer that consists in the craniocaudal direction, of the lateral epicondylopatellar band, the transverse ligament, and the patellotibial band [1]. On ultrasonography (US), the LPR was described as a thin bilayered fibrous structure [1]. Recently, a focal defect of the LPR was reported by Kim et al. [2] as a relatively common finding in patients without history of previous knee trauma or surgery, with a frequency of $26 \%$ noted on retrospective assessment of knee MRI. In the same way, the focal defect of the LPR was recently described as a route of Hoffa's fat pad herniation [3-7] as well as a route of superficial extension of infrapatellar fat lesions such as a lipoma or ganglion cyst $[7,8]$. Because US offers higher 

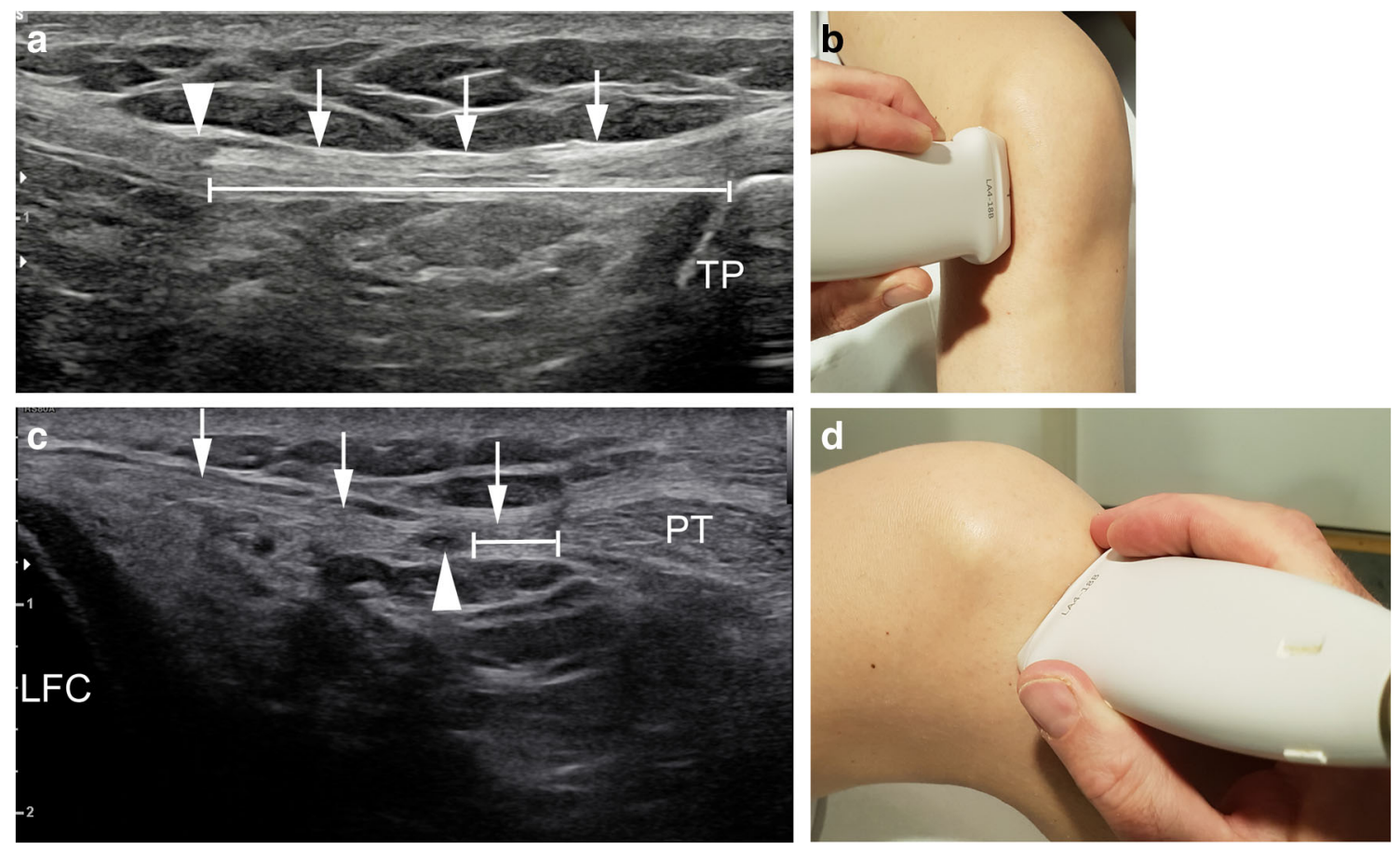

Fig. 1 Ultrasounds measurements of the defect. a Craniocaudal measurement (doubled bar line) of the defect (arrowhead) on a sagittal ultrasound image of the lateral retinaculum (arrows) in relation to the lateral tibial plateau (TP). b Corresponding position of the transducer. $\mathbf{c}$

spatial resolution than MR imaging with smaller field of view and high frequencies linear probes [9] and characterizes tissue movement in real time, the aim of our
Transverse measurement (doubled bar line) of the defect (arrowhead) on an axial ultrasound image of the right lateral retinaculum (arrows) in relation to patellar tendon (PT). d Corresponding position of the transducer

study was to evaluate whether sonography allows precise assessment of the focal defect at the LPR and to determine its anatomical significance.
Fig. 2 Cadaver dissection of a right knee confirming that the structure identified on US that was injected with China ink (arrow) (a) did represent a LPR defect related to a perforating artery (arrowheads) arising from the lateral superior and inferior genicular arteries $(\mathbf{b})(P$ patella; $T T$ tibial tuberosity)
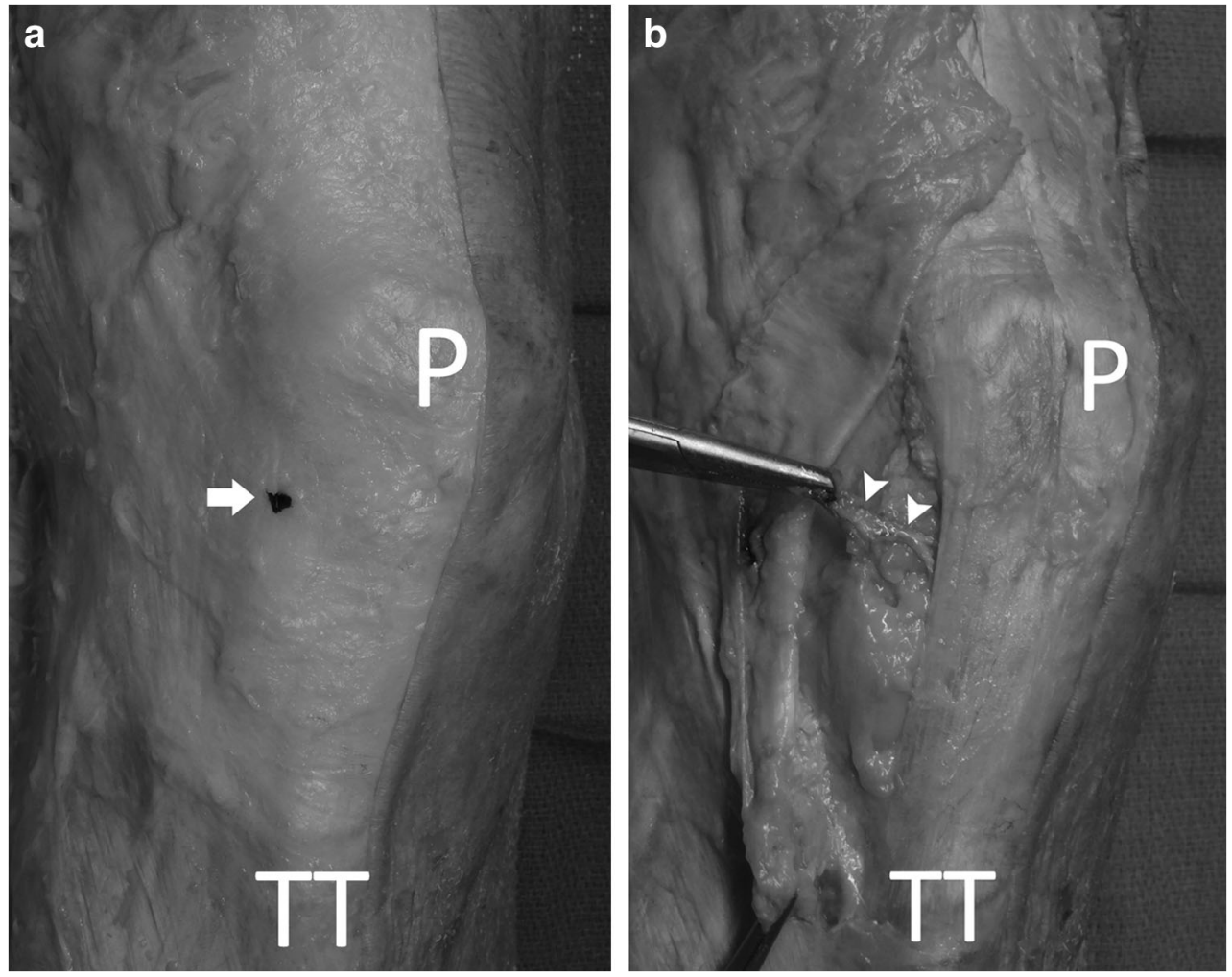


\section{Materials and methods}

\section{Anatomical study in cadavers}

In compliance with institutional safety and ethics regulations, this study was first undertaken on four formalin-embalmed cadaveric lower limbs amputated at mid-thigh level (one male and one female; mean age, 82 years) to preliminary assess the US depiction of the LPR defect and gain a better understanding of its anatomical significance. None of the cadavers showed prior evidence of knee injury or surgical procedures. The specimens were placed in supine position with the knee positioned at approximately $30^{\circ}$ of flexion and were examined using an iU22 (Philips) and a linear $17-5 \mathrm{MHz}$ probe. A staff radiologist with 13 years of experience performed all the examinations (TLC). The presence, number, and width in the axial plan of the LPR defect were recorded. The craniocaudal position of the defect was assessed in the relation to the lateral tibial plateau (Fig. 1a), the probe parallel to the lateral edge of patellar tendon (Fig. 1b). The horizontal position of the defect was measured in relation to the patellar tendon (Fig. 1c), the probe positioned in the axial plan at $90^{\circ}$ of the previous measurement (Fig. 1d). The content of the defect and its relations to adjacent anatomical structures were analyzed. Then, Indian black ink was injected under US guidance using a $25-\mathrm{G}$ needle in contact with the presumed LPR defect. Finally, a cadaver dissection was performed by an anatomist (TLC) to validate the US data. A skin and subcutaneous flap was reflected from the lateral femoral epicondyle to the medial aspect of the patella (Fig. 2). The presence, number, width in the axial plan, and craniocaudal and axial positions of the LPR defect were measured.

\section{Ultrasound study in volunteers}

Forty-eight junior elite basketball players (96 knees) of the national federal basket ball center, 23 men and 25 women, age range $16-18$ years (mean age: 17,3 years old, mean height: 190,5 cm), followed-up in a prevention protocol of jumper's knee took part in this study, which was approved
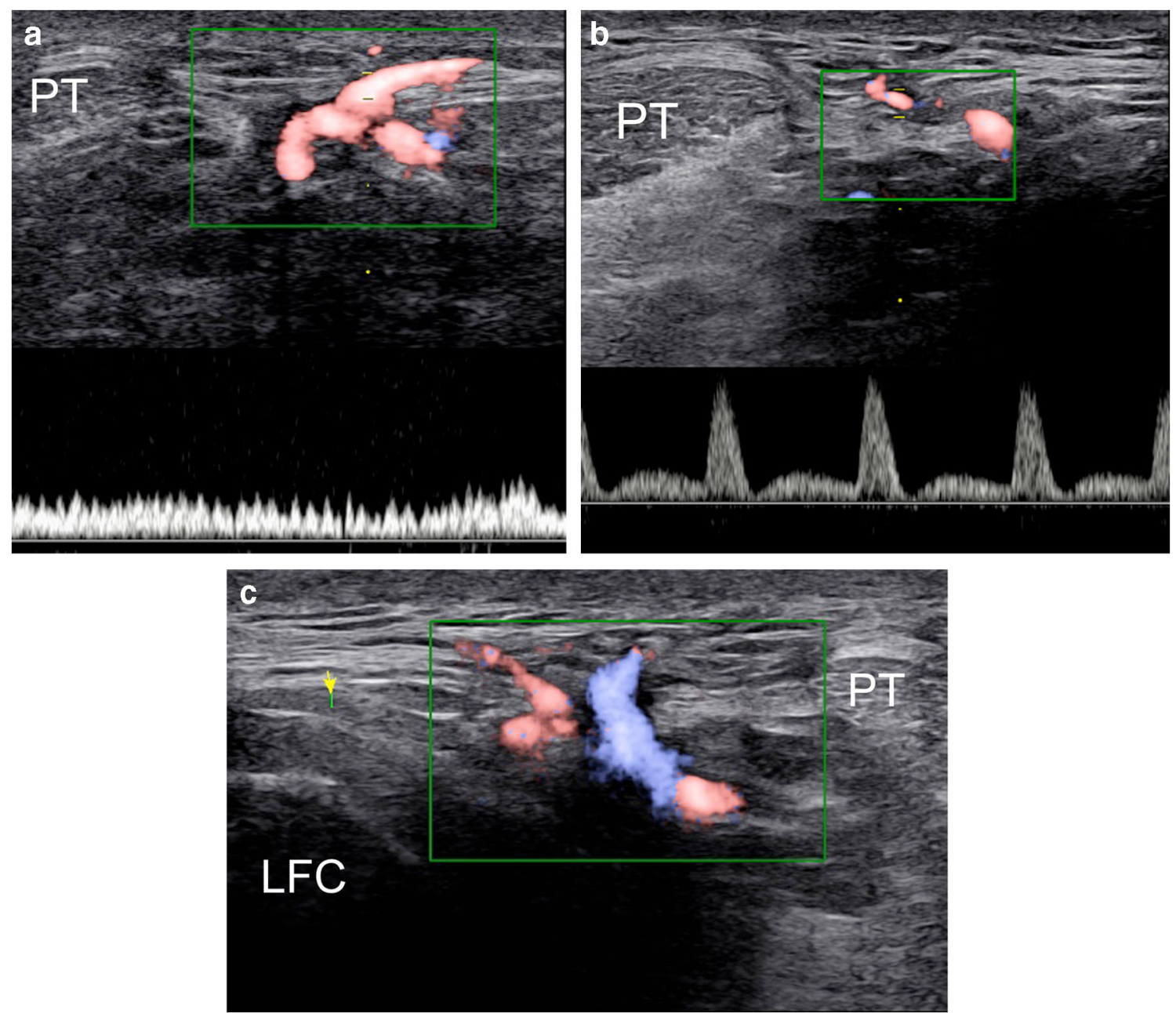

Fig. 3 Axial ultrasound image of defects of the LPR with color Doppler and pulsed Doppler showing a vein (a), an artery (b), and an artery (red) and a vein (blue) (c) passing through the defect on three different knees ( $P T$ patellar tendon; $L F C$ lateral femoral condyle) 
by our local Ethics Committee Review Board. Each participant provided informed consent. The volunteers had no history of trauma or knee surgery. All the US examinations were performed bilaterally by a second staff radiologist with 11 years of experience (AM), using a 4-18-MHz linear transducer (RS 85, Samsung Medical). The volunteers were in supine position, with the knee positioned at $35^{\circ}$ degrees of flexion on a foam positioner, then in full flexion (e.g., heels to buttocks) searching for Hoffa's fat pad herniation. The iliotibial tract delineated the area of interest laterally, the patellar tendon medially, the superior border of the lateral retinaculum and the tibial plateau inferiorly. The previously defined presence, number, size, and position of LPR defects were recorded bilaterally. The content of the defect was then assessed with color and pulsed Doppler to characterize veins and/or arteries (Fig. 3).

\section{Statistics}

Descriptive statistics were provided depending on the nature of the considered criterion: number of observed (and missing, if any) values, mean, standard deviation, median, first and third quartiles, as well as minimum and maximum, for quantitative data; number of observed (and missing, if any) values, number and percentage of patients per class, for qualitative data. The correlation between the right and left knees on transverse diameter, vertical position, and lateral position was calculated using the Pearson correlation coefficient. Statistical analysis was performed using SAS® 9.4 software.

\section{Results}

\section{Anatomic and ultrasound study on cadavers}

The anatomical data obtained from the cadavers are shown in Table 1 . In every cadaveric specimen, the US examination identified a focal defect in the LPR with vessels passing through. The mean transverse diameter was $2.75 \mathrm{~mm}$ (range, 2-3 mm). The mean vertical position was $20.75 \mathrm{~mm}$ (range, $17-26 \mathrm{~mm}$ ) and the mean lateral position was $5 \mathrm{~mm}$ (range, 4-

Table 1 Characteristics of anatomic study

\begin{tabular}{lll}
\hline & US results & Anatomical dissection \\
\hline $\begin{array}{l}\text { Number of defects } \\
\text { Transverse diameter }(\mathrm{mm}) \\
\quad(\text { mean, (range min; max)) }\end{array}$ & $2.75(2-3)$ & 1 \\
$\begin{array}{c}\text { Vertical position }(\mathrm{mm}) \\
\quad(\text { mean, (range min; max)) }\end{array}$ & $20.75(17-26)$ & $21.75(18-28)$ \\
$\begin{array}{c}\text { Lateral position }(\mathrm{mm}) \\
(\text { mean, (range min; max)) }\end{array}$ & $5(4-6)$ & $5.25(4-6)$ \\
\hline
\end{tabular}

Table 2 Characteristics of study subjects

\begin{tabular}{lcl}
\hline Number of defect(s) & Right side & Left side \\
\hline One defect (number of knees, percentage) & $30(62.5 \%)$ & $32(66.7 \%)$ \\
Two defects (number of knees, percentage) & $16(33.3 \%)$ & $16(33.3 \%)$ \\
Three defects (number of knees, percentage) & $2(4.2 \%)$ & 0 \\
\hline
\end{tabular}

$6 \mathrm{~mm}$ ). In every case, the Indian black ink could be injected under US guidance at the level of the LPR defect, in the vicinity of the penetrating vessels (Fig. 2a). Subsequent cadaver dissection confirmed that the injected structure visualized on US in fact represented a defect within the LPR related to perforating vessels originating from the peripatellar anastomotic ring. In particular, a perforating artery arising from the lateral superior and/or lateral inferior genicular arteries was anatomically identified passing through the defect (Fig. 2b). The same mean transverse diameter, vertical position, and lateral position measured anatomically were $2.5 \mathrm{~mm}$ (range, 2-3 mm), $21.75 \mathrm{~mm}$ (range, 18-28 mm), and $5.25 \mathrm{~mm}$ (range, 4-6 mm), respectively (Table 1).

\section{Ultrasound study on volunteers}

The US data obtained in 48 volunteers ( 96 knees) are shown in Tables 2 and 3. A total number of 132 defects were depicted, 68 defects on right knees and 64 on left knees. At least one defect was depicted by knee. On right knees, one defect was depicted in 30 knees (62.5\%), two defects in 16 knees (33.3\%), and three defects in two knees (4.2\%). On left knees, one defect was depicted in 32 knees $(66.7 \%)$, two defects in 16 knees $(33.3 \%)$ (Table 2). The mean transverse diameter was $2.4 \mathrm{~mm}$ (range, min-max: $1-6 \mathrm{~mm}$ ). The mean vertical position was $23.7 \mathrm{~mm}$ (range, min-max: $18-30 \mathrm{~mm}$ ) and the mean lateral position was $6.6 \mathrm{~mm}$ (range, min-max: 4-9 $\mathrm{mm}$ ) (Table 3). The defect contents an artery in 84 defects (65.6\%), vein(s) in 15 defects (11.7\%), artery and vein(s) in 29 defects $(22.7 \%)$ (Table 3$)$. The correlation between right and left knees was weak for the diameter, for the lateral position and

Table 3 Characteristics of study subjects

\begin{tabular}{ll}
\hline Position & \\
$\quad \begin{array}{l}\text { Transverse diameter (mm) (mean; } \\
\text { median (range min; max)) }\end{array}$ & $2.4 ; 2(1-6)$ \\
$\quad$ Vertical position (mm) (mean; & $23.7 ; 26(18-30)$ \\
$\quad$ median (range min; max)) & \\
$\quad$ Lateral position (mm) (mean; & $6.6 ; 6(4-9)$ \\
$\quad$ median (range min; max)) & \\
Content & \\
Artery & $84(65.6 \%)$ \\
Vein & $15(11.7 \%)$ \\
Artery and vein & $29(27.7 \%)$ \\
\hline
\end{tabular}




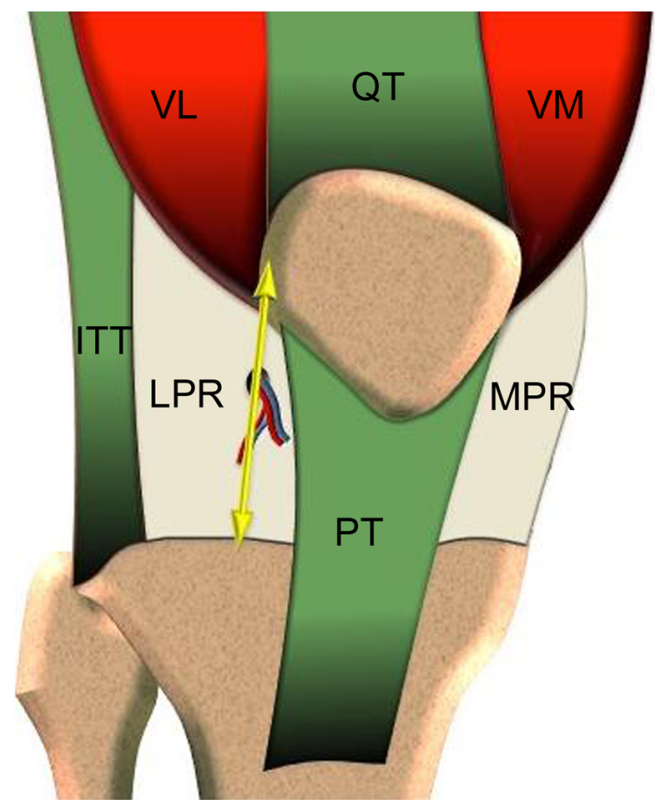

Fig. 4 Schematic representation of the vertical line position of the defect of the LPR, 5-6 mm lateral to the patellar tendon ( $L P R$ lateral patellar retinaculum, $M P R$ medial patellar retinaculum, ITT iliotibial tract, $Q T$ quadriceps tendon, $P T$ patellar tendon, $V L$ vastus lateralis, $V M$ vastus medialis)

for the vertical position $(0.43,0.42$, and 0.32 , respectively). A single asymptomatic Hoffa's fat pad herniation was depicted on the left knee of a girl through the largest large defect of the study measured at $6.15 \mathrm{~mm}$ of width at rest. The prevalence of Hoffa's fat pad herniation was $2.1 \%$ among our population.

\section{Discussion}

The LPR is one of the three main passive stabilizers of the patella in association with the medial patellar retinaculum and the patellar tendon. It is a complex anatomical structure that presents a superficial layer that extends from the lateral margin of the patella and patellar tendon to the iliotibial band and vastus lateralis muscle fascia laterally, and a deep layer containing reinforcements that form ligaments providing stabilizing support to the patella. This deep layer consists, in the craniocaudal direction, of the lateral epicondylopatellar band, the transverse ligament, and the patellotibial band. The transverse ligament is $1-2 \mathrm{~cm}$ in width and extends from the deep portion of the iliotibial tract to the lateral patellar margin. The lateral epicondylopatellar band arises from the patella above the transverse ligament and inserts into the lateral epicondyle and intermuscular septum. The patellotibial band arises from the patella below the transverse ligament and inserts into the proximal tibia and lateral meniscus [1]. Kim et al. [2] recently demonstrated in an anatomical study that the LPR is more loose and sparse than the medial patellar retinaculum and showed that this site corresponds to the LPR defect on MR imaging. Therefore, these authors suggested that a focal defect of the LPR may be a normal variant rather than a pathologic change because of trauma or degeneration. On the other hand, high-resolution US, because it offers several advantages over other imaging modalities, has become an attractive complement for assessing thin and superficial anatomical structures in the musculoskeletal system. As no description of the LPR defect by means of sonography has been reported, the aim of the present study was to evaluate whether US allows precise assessment of the defect and to determine its anatomical significance. In our practice, a focal defect at the LPR through which vessels penetrate is a very common asymptomatic finding during routine US examinations, suggesting that the defect is a normal vascular channel rather than a variant. In addition, we previously reported cases of Hoffa's fat pad herniation and a case extrusion of a lipoma and of a ganglion cyst through such defects, which supports that defects of the LPR are anatomically a route of superficial extension of infrapatellar fat lesions such as a lipoma or ganglion cyst [7, 8]. Our results obtained in cadavers show that a focal defect of the LPR through which vessels penetrated could be constantly identified by ultrasound. The LPR defect was relatively small and was located along a vertical line approximately $5 \mathrm{~mm}$ lateral to the patellar ligament between 17 to $26 \mathrm{~mm}$ proximal to the lateral tibial plateau. Furthermore, anatomical dissection confirmed that the LPR defect was related to perforating arteries and/or veins that originate from the peripatellar
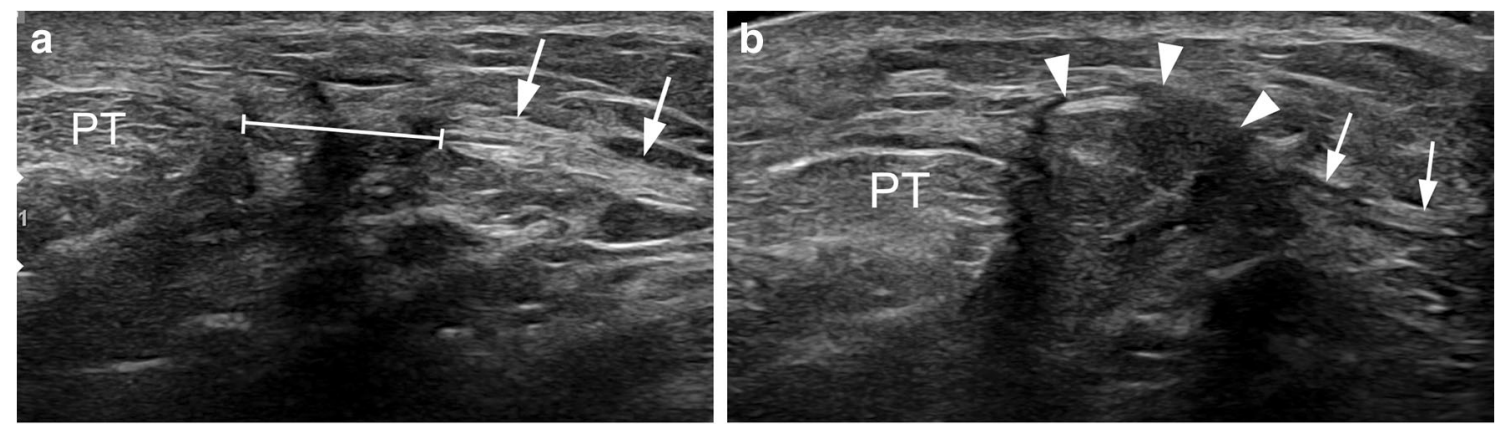

Fig. 5 Axial ultrasound image at rest of the largest defect (doubled bar line) of the LPR (arrows) in the left knee (a) (PT patellar tendon), and in maximal flexion showing an extrusion of the Hoffa's fat pad through the defect of the LPR (b) (PT patellar tendon) 
anastomotic ring. In the US study on volunteers, LPR defects were found to be constant, sometimes even multiple, and were smaller than reported by Kim et al. in a previous MR study. Hence, the mean transverse diameter of the defect in our population was $2.4 \mathrm{~mm}$ versus $4.9 \mathrm{~mm}$ in the study by Kim et al. Interestingly, the vertical position of the defects was highly variable, ranging from 18 to $30 \mathrm{~mm}$ with no correlation between right and left knees, whereas the lateral position was more constant with a mean of $6.6 \mathrm{~mm}$ versus $2 \mathrm{~mm}$ in the study by Kim et al. On a schematic representation, the defects were located at various heights on a vertical line drawn at about 5 to $6 \mathrm{~mm}$ lateral to the patellar tendon (Fig. 4). These differences of size and position may be due to the comparison of two different imaging modalities with higher spatial resolution and smaller fields of view in US than MRI, and with local compression with the US probe. Color and pulsed Doppler study in volunteers showed that arteries, veins or both pass systematically through the LPR defect which is concordant with our anatomical observations and with the histological findings of Kim et al. Dynamic ultrasound disclosed a single asymptomatic herniation of Hoffa's fat pad through the largest defect observed in our volunteer population (Fig. 5a). This herniation was only depicted with US in full flexion (Fig. 5b) with no spontaneous clinical lump, probably because the herniation may be hidden by the superficial soft tissue layer of the knee, which is thicker in adults than in children. These hernias may therefore be more frequent than previously reported. Our study presents several limits. First, we dissected only four cadaveric specimens, which is relatively small for an anatomical study. Second, we did not analyze the intra- or interobserver variation in the US study. The preliminary assessment of the LPR defect undertaken in cadavers and the US examinations in healthy volunteers were instead performed by two experienced operators independently. Finally, the results observed in our volunteer population might have been influenced by the elevated mean height of these junior basketball players, which is why we present in the abstract of this study the results obtained in cadavers and confirmed by direct anatomical measurement.

In conclusion, our study has demonstrated that a focal defect of the LPR through which small artery and/or vein penetrated could constantly be identified by US. The LPR defect is usually located 5-6 $\mathrm{mm}$ lateral to the patellar tendon, and between 17 and $30 \mathrm{~mm}$ proximal to the lateral tibial plateau. Large LPR defects may be a route for extrusion of Hoffa's fat pad. Such hernias may be asymptomatic with no clinical lump and may be more frequent than previously reported.

\section{Compliance with ethical standards}

Guarantor The scientific guarantor of this publication is Thomas Le Corroller, M.D., Ph.D.

Conflict of interest The authors of this manuscript declare no relationships with any companies, whose products or services may be related to the subject matter of the article.

Statistics and biometry Hugo Caillou from Capionis kindly provided statistical advice for this manuscript.

Informed consent Written informed consent was obtained from all subjects (patients) in this study.

Ethical approval Institutional Review Board approval was obtained.

Study subjects or cohorts overlap No study subjects or cohorts have been previously reported.

Methodology Methodology: prospective.

\section{References}

1. Starok M, Lenchik L, Trudell D, Resnick D. Normal patellar retinaculum: MR and sonographic imaging with cadaveric correlation. AJR Am J Roentgenol. 1997;168(6):1493-9.

2. Kim JS, Yun SJ, Jin W, Kim GY, Park SY, Park JS, et al. A focal defect at the lateral patellar retinaculum on clinical knee MRI and cadaveric study: a normal variant or pathologic lesion? AJR Am J Roentgenol. 2017;208(5):1103-9.

3. Grobbelaar N, Bouffard JA. Sonography of the knee, a pictorial review. Semin Ultrasound CT MR. 2000;21(3):231-74.

4. Park SY, Jin W, Rhyu KH, Park JS. Herniation of infrapatellar fat through defect of lateral retinaculum in preschool age children: imaging findings on ultrasonography. Electronic Presentation Online System: European Society of Radiology; 2010.

5. Rocha D, Ramos R, Campos J, Pinto D, Pimenta M. Focal herniation of Hoffa's fat pad through a retinaculum defect. Radiological Case Database: EuroRad; 2011.

6. Chauvin NA, Khwaja A, Epelman M, Callahan MJ. Imaging findings of Hoffa's fat pad herniation. Pediatr Radiol. 2016;46(4):508-12.

7. Moraux A, Bianchi S, Corroller L, Soft T. Tissue masses of the knee related to a focal defect of the lateral patellar retinaculum. J Ultrasound Med. 2018;37(7):1821-5.

8. Saha P, Bandyopadhyay U, Mukhopadhyay AS, Kundu S, Mandal S. Ganglion cyst of knee from Hoffa's fat pad protruding anterolaterally through retinacular rent: a case report. J Orthop Case Rep. 2015;5(3): 69-71.

9. Alves TI, Girish G, Kalume Brigido M, Jacobson JA. US of the knee: scanning techniques, pitfalls, and pathologic conditions. Radiographics. 2016;36(6):1759-75. 